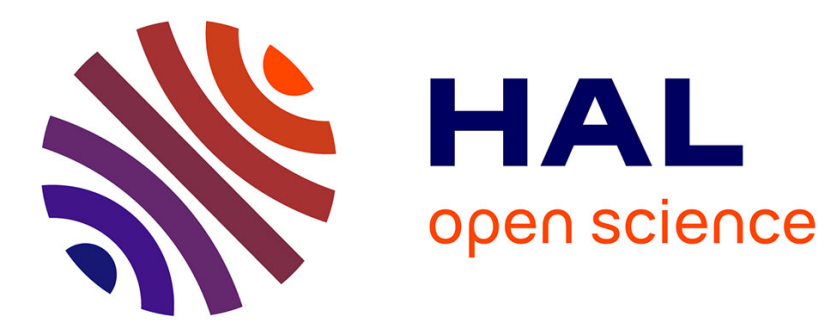

\title{
ESR: Energy aware and Stable Routing protocol for WBAN networks
}

\author{
Omar Smail, Adda Kerrar, Youssef Zetili, Bernard Cousin
}

\section{To cite this version:}

Omar Smail, Adda Kerrar, Youssef Zetili, Bernard Cousin. ESR: Energy aware and Stable Routing protocol for WBAN networks. 12th International Wireless Communications \& Mobile Computing Conference (IWCMC 2016), Sep 2016, Paphos, Cyprus. 10.1109/IWCMC.2016.7577100 . hal-01321647

\section{HAL Id: hal-01321647 \\ https://hal.science/hal-01321647}

Submitted on 26 May 2016

HAL is a multi-disciplinary open access archive for the deposit and dissemination of scientific research documents, whether they are published or not. The documents may come from teaching and research institutions in France or abroad, or from public or private research centers.
L'archive ouverte pluridisciplinaire HAL, est destinée au dépôt et à la diffusion de documents scientifiques de niveau recherche, publiés ou non, émanant des établissements d'enseignement et de recherche français ou étrangers, des laboratoires publics ou privés. 


\title{
ESR: Energy aware and Stable Routing protocol for WBAN networks
}

\author{
Omar Smail ${ }^{1}$, Adda Kerrar ${ }^{1}$, Youssef Zetili ${ }^{1}$, Bernard Cousin ${ }^{2}$ \\ ${ }^{1}$ Faculty of exact sciences. Computer Science Department. \\ University Mustapha Stambouli, Mascara, Algeria. \\ ${ }^{2}$ IRISA/University of Rennes 1 , France. \\ smailomar@ieee.org, \{a.kerrar, y.zetili\}@univ-mascara.dz, bcousin@irisa.fr
}

\begin{abstract}
Wireless body area network (WBAN) calls for a next generation in wireless networks. This new generation is designed to operate autonomously, to connect various medical sensors and appliances located on or inside a human body. Mobile WBANs have been designed, offering numerous practical and innovative services so that health care and quality of life can be improved. Thus, the equipment used in WBAN is usually mobile and autonomous which imposes high constraint on energy. That is, the energy efficiency must be taken into account as one of the objectives of the routing protocol designed for this type of network. Although mobile nodes may cause link breaks, most of studies ignore the link stability. In this paper, we propose a stable, reliable, energy efficient routing protocol for mobile Wireless Body Area Networks. It preserves the residual energy of nodes with an increase network lifetime. To achieve this goal, we use an objective model to select energy-efficient paths with stable links. Simulation results demonstrate that our protocol improves the state of the art in terms of energy consumption and routing overhead.
\end{abstract}

Keywords-Mobile WBAN network; Body area network; Link stability; Energy efficiency

\section{INTRODUCTION}

A wireless Body Area Network (WBAN) is a radio frequency based wireless networking technology that interconnects nodes with sensor or actuator capabilities in, on, or around a human body. These nodes are body sensors that can monitor the human body functions and characteristics of its surrounding environment. The main applications of WBANs are in the areas of health, first aid, military, entertainment, sports, ambient intelligence and human-to-machine interactions. The introduction of WBAN into medical monitoring and other health applications offer flexibilities and cost saving options to both health care professionals and patients. A WBAN system can offer two significant advantages compared to current electronic patient monitoring systems. The first advantage is that it permits the mobility of patients with the use of portable devices. The second advantage is the location independent monitoring facility, that is, being an autonomous device; a mobile WBAN node can search and find a suitable communication network to transmit data to a remote database server for storage. It can also connect itself to the internet for the transmission of data. The body sensors are installed on or inside the human body to communicate with a hub device, which is located on or near the individual.
The body sensor readings of the human body's vital signs such as temperature, blood pressure and ECG (electrocardiogram) are sent to the hub device. This hub device may in turn communicate to others via another longer-range network. In BAN, the body sensors generally send their data to a body coordinator. The body coordinator behaves as a router in BAN networks and communicates with other devices. These devices are mobiles and can be used to transmit information to (resp. from) the human body to (resp. from) the external world (physician, emergency). In order to establish communication between two of these devices, routing techniques from Wireless Sensor Networks and ad hoc networks could be used. The communication architecture for these devices can be centralized or distributed. However, because of theirs typical properties, current routing protocols designed for these networks are not always well suited to support a WBAN. Designing such protocols implies challenges including the combination of path-reliability, short-range transmission, low data rate, low energy consumption, body mobility, and transmission security [1-5]. In particular, energy efficiency constitutes a significant factor for longterm deployment of WBANs, addressed by many works [610]. The class of wireless body network sets additional issues: the stability of the links. The residual energy of the nodes, their mobility and the link overload are the main issues that impact the stability of the links.

In this paper, we propose a novel routing protocol for WBAN, called ESR (Energy aware and Stable Routing protocol). ESR protocol is designed for mobile nodes with power limited, where link failures may occur frequently. For this purpose, we suggest a novel formulation of the WBAN energy efficient communication problem, while taking accurate account of link stability issues. The main performance of this protocol is to select the links that ensures the lowest energy consumption with stable links. We combine energy efficiency and stability of links into an objective function. Our goal is to improve the WBAN network performance by reducing the energy consumption and maximize network lifetime. Additionally, the proposed solution should not generate too much control messages because we are in a type of low-throughput network.

The paper is organized as follows: Section 2 provides a review of related works for energy aware routing protocol in wireless body area networks. Section 3 describes the different WBAN communication architectures. Section 4 
gives the design details of our ESR protocol. Section 5 provides simulation results of its performance evaluation. Section 6 concludes this work.

\section{RELATED WORK}

In WBAN domain, a large numbers of routing schemes have been proposed. They consider various objectives. In this section, we present the main energy aware routing protocols. Traditionally, there are two routing approaches. One approach is to integrate the routing functions with the MAC layer using a fundamental cross-layer approach. This approach allows the use of link quality parameters but with an increase of the complexity. The second approach is to design a routing layer on top of the MAC layer, where link qualities are measured based on few selected parameters managed at the routing layer. With thus second approach, the two layers are separate, thus the complexity is low. The SIMPLE protocol for WBAN is introduced in [11]. In this protocol, a cost function is used to select the parent node, or forwarder, with high residual energy and minimum distance to the sink. Nodes with less value of cost function are elected as parent node. Residual energy parameter balances energy consumption among the sensor nodes while distance parameter ensures successful packet delivery to sink. Two nodes for ECG and Glucose level are placed near the sink. Both sensors generate critical data for the patient and require minimum attenuation, high reliability and long life. Therefore, these sensors always transmit their data directly to the sink. Other sensors follow their parent node and transmit their data to the sink through forwarder node. The simulation results show that proposed routing schemes enhance the network stability time and packet delivered to the sink.

In [12], a protocol, named Co-CEStat for Wireless Body Area Networks, is proposed. The protocol utilizes the merits of both direct and cooperative transmissions to achieve higher stability period and end-to-end throughput with greater network lifetime. Co-CEStat uses a multi-tier architecture in which nodes are affixed to the body of the patient and the sink is placed at the center of the body. The proposed protocol is an energy efficient routing protocol, which utilizes dynamic routing with cooperation between nodes for data forwarding. It exploits cooperative routing in heterogeneous network to enhance WBAN performance. By avoiding redundant data transmission, Co-CEStat is efficient in terms of energy conservation and overall network throughput compared to other protocols.

A cooperative routing protocol which considers energy consumption and QoS as performance parameters is proposed in [13]. QoS is measured by the absolute received signal strength indicator (RSSI). To integrate consumption and QoS parameters in the routing protocol, a competitive/opponent mechanism is implemented at each node by utilizing multi-agent reinforcement-learning algorithm. The proposed algorithm (RSSI/energy-CC) is an energy and QoS aware routing protocol since it ensures good performance in terms of end-to-end delay and packet loss rate, taking into account the consumed energy through the network. Cooperative communication allows sharing of resources, thereby, enhancing the network performance. The protocol simulations show that the protocol is efficient in terms of percentage of lost packets, network energy consumption, maximal energy consumption per node, and network lifetime.

In [14], a Minimum Energy Packet Forwarding Protocol (MEPF) is designed. Firstly, it considers minimum energy packet forwarding as a whole, by identifying the trade-off of the transmission power control saving and the retransmission energy consumption. Secondly, it proposes lightweight solutions for transmission power control and ACK power control as smart ARQ (Automatic Repeat request). MEPF transmits a packet using the minimum transmission power that can guarantee a high packet reception rate. At the same time, retransmissions are postponed until the link is good enough. The quality of the link is dynamically judged by a machine learning algorithm, designed for the limited capabilities of typical Body Area Sensor Networks devices. Through MEPF, the energy consumption to forward a packet is minimized without sacrificing the packet reception ratio.

Maskooki et al. [15] observe that the body movement could degrade the network performance in WBANs. Nodes mobility could pose a problem especially in energy efficiency. Therefore, an opportunistic routing is proposed to overcome this body movement problem. Actually, the idea of this protocol is simple; it is how to transmit data in LOS (Line of Sight) condition as long as possible. The idea could be realized by using a relay node located at a right place. In this proposal, it is assumed that the sink node is located on wrist and a sensor node is placed on chest. While the person is walking, his hand will go back and forth so sometimes the sensor node will transmit a LOS signal (when the wrist is in front of the body), while at other times; the signal is a NLOS (when the wrist is behind the body). When the hand node is in LOS position, the sensor node will transmit the data directly to sink node, while when the hand node is in NLOS position, the sensor node will make use of relay node - which is in LOS position to both sink and sensor node - to send data to the sink node. The sensor node will opportunistically use the relay node to be able to transmit data in LOS condition as long as possible.

A protocol, named Distance Aware Relaying Energy Efficient (DARE) to monitor patients in multi-hop Body Area Sensor Networks (BASNs), is provided in [16]. To decrease the energy consumption, the sensors creates a link with the sink by means of an on-body relay, attached on the chest of each patient. The attached body relay retains greater energy resources as compared to the body sensors, since they perform gathering and relaying of data to the sink node. Hence, for heterogeneous networks (for instance for monitoring patients), a relaying energy-efficient protocol is suggested. In the given scenarios, few sensors nodes constantly monitor patient's data whereas some other nodes monitor data only when they notice a definite threshold stage. The protocol defines minimum energy parameters for the sensors to avoid heat damage to the sensitive human body tissues. Results of the research depict greater packet delivery ratio, longer network life time and better stability period. However, it has high propagation delay.

In [17] an improved version of Power-Efficient Gathering in Sensor Information Systems (PEGASIS) is presented. This paper introduces mobility of the sink and extends the network lifetime of Wireless Sensor Networks 
(WSNs). The concept of multi-head chain, multi-chain and the sink mobility greatly increase the network lifetime of wireless sensor nodes. The recommended concept of mobile sink improved energy-efficient PEGASIS-based routing protocol (MIEEPB),-MIEEPB is a multi-chain model, which provides mobility of sink, to attain effective energy usage of wireless sensors. There is a need to enclose its movement within boundaries and the route of mobile sink should be static as the automated shifting of mobile sink is steered by petrol or current. The outcomes of this research indicate that the proposed scheme is efficient in terms of network lifetime and stability period. It controls the average energy consumption of nodes, and shrinks the delay in data delivery by means of smaller chains.

The work in [18] proposes a novel BAN architecture for indoor hospital scenario, and a new Energy aware Peering Routing protocol (EPR), which includes a new mechanism of peer discovery with routing table construction that helps to reduce network traffic load, energy consumption, and improves BAN reliability. The proposed BAN peering framework and routing protocol are designed to display in real-time BAN data, avoid a fully centralized system, and discover the dedicated BAN data display unit dynamically. Both centralized and distributed approaches are used in the proposed scheme. Only the central computer holds the information of BANs and display units, which helps to improve privacy and better control BAN communication. The BAN data is displayed on the display unit in a distributed manner, which reduces traffic load and helps to improve patient mobility. All of the above studies solve the problem of energy consummation by adjusting the forward transmission and also the ACKs, but the majority of powersaving mechanisms are based only on the remaining power cannot be used to establish the best route between source and destination nodes. On one hand, the majority of energy efficient protocols for mobile WBANs, select the next hop following energy consideration of the node, but if a node is willing to accept all route requests only because it currently has enough residual battery capacity, too much traffic load may be routed through that node. In addition energy is not the only factor since the nodes can be mobile which cause their disconnection even if they have enough energy, so the study of their stability is essential. On the other hand, these protocols are based on the selection of one hop to reach the destination, while several nodes (multi-hop) may cooperate to reach the destination. Finally, the majority of these protocols have been compared only with their original protocols, or protocols which do not explicitly consider energy consumption, and thus these performance evaluations are not fair.

\section{BAN COMMUNICATION ARCHITECTURE}

In a BAN, the body implants and wearable sensors send their data to a central device known as the body coordinator. Then, through a WLAN connection, these data are streamed remotely to a medical doctor's site for real time diagnosis, to a medical database for record keeping, or to the corresponding equipment that issues an emergency alert. Thus, we define a BAN architecture with a three Tier design: Tier-1 communications (i.e., intra-BAN communications), Tier-2 Communications (i.e., inter-BAN communications,), and Tier-3 communications $n$ (i.e., beyond-BAN communications) [19]. The intra BAN communications works into the BAN of a person and runs between a body sensor of this person and its body coordinator. Inter BAN communications run between one or several body coordinators and a static coordinator. The static coordinator runs as an Internet gateway and is able to command a set of body coordinators in order to control their personal-BAN. An inter BAN communication may be a centralized BAN communication and a distributed BAN communication, see Figure 1.
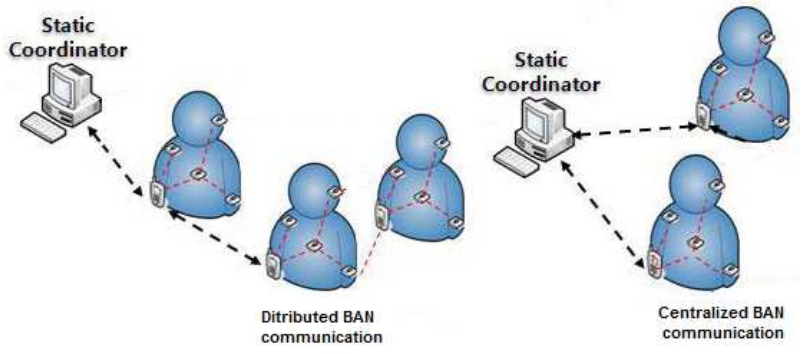

Figure 1: Centralized and distributed BAN communications

In a centralized BAN communication, the static coordinator is directly connected with each body coordinator. In a distributed BAN communication, data transmission between a body coordinator and the static coordinator may run through intermediate body coordinators. Centralized communications are easier to manage and produce low transmission delay. However when the body coordinator is out of reach of the static coordinator, centralized communications cannot be established. Distributed communications introduce flexibility and increase communication reach.

\section{ENERGY AWARE AND STABLE ROUTING PROTOCOL}

The main objective of this paper is to develop an improved routing protocol, named Energy aware and Stable Routing protocol (ESR), for WBAN networks. The ESR protocol is intended to be employed in hospital environment for body networks mainly in a distributed communication. We use an objective model to select energy-efficient paths with stable links, in order to reach the static coordinator. In this section, we provide the details of the discovery and selection of the best path.

\section{A. Path discovery process}

Based on the on-demand routing scheme, the source BAN coordinator starts the path discovery process to create a set of paths able to forward data towards the destination static coordinator from the source BAN coordinator.

When a source BAN coordinator requires a path toward a destination static coordinator, the source BAN coordinator checks its routing table for any available path toward this destination static coordinator. If a path is not present or is invalid, the source BAN coordinator performs route discovery: it broadcasts an RREQ (Route Request) message to all of its neighbors. When an intermediate BAN coordinator node receives an RREQ, it ensures that the received RREQ is not a duplicate RREQ by comparing the RREQs' identifiers, in order to prevent looping paths. Otherwise, the RREQ-receiving nodes verify whether they have any valid path toward the destination static coordinator in their routing tables. If they have, they forward the RREQ to those valid path neighbors. In this 
case, for our protocol, returning back a RREP (Route Reply message) to the source BAN coordinator is not desirable because we would like to collect fresh energy information. Otherwise, the receiving BAN coordinator node retransmits the RREQ message to all of its neighboring nodes to find the paths toward the destination static coordinator. When the destination static coordinator receives the first RREQ, it waits for a certain time (RREQ_Wait_Time) and collects all other RREQs arriving during this time interval. This waiting period is necessary to receive the RREQ packets, and before it expires, the destination static coordinator must have answered by sending an RREP message towards each node from which it received an RREQ message. When a BAN coordinator node receives its first RREP message, it creates a path entry towards the node from which it received the RREP message and updates its routing table. When the source BAN coordinator receives the first RREP message, it waits for a certain amount of time (RREP_Wait_Time) to receive more RREPs before selecting the best path.

\section{B. Path routing selection}

This section describes path selection routing. The choice of the best path between a source BAN coordinator node $s$ and destination static coordinator node $d$, is done according to energy consumption and path stability. Here, two functions are defined: the cost function $f e p_{j}(t)$, which characterizes a path $j$ at time $t$ from an energetic point of view and the cost coefficient $f s p_{j}(t)$, which represents the stability of path $j$ at time $t$.

\section{1) Energy aware function}

Let $f e p_{j}(t)$ be the minimum residual energy of nodes constituting the path $j$ for a source BAN coordinator node $s$ to destination static coordinator node $d$ at time $t$ :

$$
f e p_{j}(t)=\min _{i=1}^{n-1}\left(\mathrm{fen}_{i, j}(t)\right)
$$

Where $f e n_{i, j}(t)$ represents the energy cost function of node $i$ belonging to the path $j$ :

$$
\operatorname{fen}_{i, j}(t)=\frac{\operatorname{Elev}_{i, j}(t)}{D R_{i, j}(t)} \cdot w_{i, j}
$$

$\operatorname{Elev}_{i, j}(t)$ denotes the energy level of node $i$ belonging to the path $j$ at time $t$ during a discovery process between a source BAN coordinator node $s$ and a destination static coordinator node $d$, given by:

$$
\operatorname{Elev}_{i, j}(t)=\frac{E_{i, j}(t)}{E_{\text {average }}}
$$

Where $E_{i, j}(t)$ represents the node $i$ residual energy belonging to the path $j$ at time $t$ and $E_{\text {average }}$ is the average residual energy of nodes that participated in the path discovery process between one source BAN coordinator node $s$ and one destination static coordinator node $d$. $w_{i j}$ : weight factor of node $\mathrm{i}$ belonging to the path $j$, which depends on various factors, like battery's quality, battery's capacity, life time, and battery's back up. $D R_{i j}(t)$ is the drain rate of the node $i$ belonging to the path $j$ at time $t$, which is defined as the rate at which energy is consumed at a given node, usually when a node is used by paths for others of our source BAN coordinator $s$ and destination static coordinator $d$.

\section{2) Link stability aware function}

To calculate the link stability in mobile WBAN networks, we consider the mobility of nodes as the main metric. The protocols based on the parameters of nodes' mobility use some criteria inherent of the nodes mobility, such as their coordinates, their directions of movement or their speeds, into the calculation of the stability metric values. We use the node coordinates for our protocol. We exploit discoveries messages and hello messages for collecting coordinate information of neighbors nodes, to avoid the overcost of new send specific messages to collect these nodes information's coordinates. Figure 2 shows the structure of an entry of the routing table of a node $i$. For each destination static coordinator known by the node $i$ there is an entry. Route_list contains all known neighboring nodes of a node $i$ which leads to that destination static coordinator. Each neighbor for that destination static coordinator is identified its nexthop address, and the hopcount field is the number of hops required to reach that destination static coordinator using this neighbor. We add two new fields compared the basic routing table, $E_{i}$ and distances_list. The field $E_{i}$ denotes the residual energy of a node $i$ and the field distances_list indicates registered distances between the node $i$ and its neighbors over different time.

\begin{tabular}{|l|}
\hline Destination \\
\hline Sequence_number \\
\hline Advertised_hopcount \\
\hline Route_list \\
$\left\{\left(\right.\right.$ nexthop1, hopcount $1, \mathrm{E}_{1}$, \\
distances_list $\left.\left\{\left(\mathrm{d}_{\mathrm{i} 1}, \mathrm{t}_{1}\right),\left(\mathrm{d}_{\mathrm{i} 1}, \mathrm{t}_{2}\right), \ldots\right\}\right)$, \\
(nexthop2, hopcount $2, \mathrm{E}_{2}$, \\
distance_list $\left.\left.\left\{\left(\mathrm{d}_{\mathrm{i} 2}, \mathrm{t}_{1}\right),\left(\mathrm{d}_{\mathrm{i} 2}, \mathrm{t}_{2}\right), \ldots\right\}\right), \ldots\right\}$ \\
\hline Expiration timeout \\
\hline
\end{tabular}

Figure 2: Structure of a routing table entry for ESR.

Assuming a link between two nodes $i$ and $k$, we calculate the stability of this link. The node $i$ periodically sends message to the node $k$, when the node $k$ receives the message, detects its coordinates then sends it to the node $i$. Based on this information, the node $i$ calculates the distance that separates the node $k$ noted by $d_{i, k}$. The stability cost function of the link $i, k$ at time $t$, is denoted by: $f s l_{i, k}(t)$, given by:

$$
f s l_{i, j}(t)=\frac{S D l_{i, k}(t)}{M l_{i, k}(t)}
$$

Where $M l_{i, k}(t)$ represents the mean of the $m$ distances recorded between the node $i$ and node $k$, defined as follows:

$$
M l_{i, k}(t)=\frac{\sum_{t=t 1}^{t m} d_{i, k}(t)}{n}
$$

$S D l_{i, k}(t)$ denoted the standard deviation of the distances recorded between the node $i$ and node $k, S D l_{i, k}(t)$ is given by:

$$
S D l_{i, k}(t)=\sqrt{\frac{1}{n} \sum_{t=t 1}^{t n}\left(d_{i, k}(t)-M l_{i, k}(t)\right)^{2}}
$$

The function $f s l_{i, k}(t)$ represents the coefficient of variation, also known as relative standard deviation. It is a standardized measure of dispersion of a probability distribution or frequency distribution. The coefficient of 
variation formulated by $f s l_{i, k}(t)$ is used to quantify the measurement accuracy. In our case, it is the measurement of distances between two neighboring nodes. If the $f_{s} l_{i, k}(t)$ tend to 0 , then we have a good distribution of distances, which means that the link is stable. If it tends to 1 or to infinity, this corresponds to a poor distribution, which means that the link is instable.

Finally, we define $f s p_{j}(t)$ the path cost function stability, given by:

$$
f_{s p}(t)=\max _{i=1}^{n-1}\left(f s l_{i, k}(t)\right)
$$

With $n$ defined as the nodes number of the path $j$ and $k$ as the neighbor node of the node $i$. The path cost function stability is based on the cost function stability of the links constituting this path. $f s p_{j}(t)$ is defined as the maximum stability cost of links constituting the path $j$.

\section{3) Objective problem formulation}

We design our path selection principle on the ordering of paths according to the energy consumption of their path nodes and the link stability of their path links. To satisfy this, we use a model using arbitrary importance weights for each criterion $(\alpha$ and $\beta$ ). The corresponding objective function $f p_{j}(t)$ is defined by combining the formulas (1) and (7):

$$
f p_{j}(t)=\alpha f e p_{j}(t)+\beta / f s p_{j}(t)
$$

Our idea is based on sorting all paths between a source BAN coordinator node $s$ and destination static coordinator node $d$ by the descending value of $f p_{j}(t)$. The path with the maximum $f p_{j}(t)$ is chosen to forward the data packets. We note that our model can be used to address many applications, with different constraints when we choose appropriate values for the parameters $\alpha$ and $\beta$. For instance, with applications that require energy saving, more importance is given to the coefficient $\alpha(\alpha>>\beta)$, since it is the weight associated to the path energy in the model. Finally, we note that the weights $\alpha$ and $\beta$ are chosen such that the condition $\alpha+\beta=1$ is satisfied.

\section{Performance EVAluation of ESR}

In this section, we present simulation results to demonstrate the efficiency of our proposed protocol. First we present the metrics used for performance evaluation and then we evaluate our protocol by comparing it with the protocol EPR [18]. We will use EPR as a reference for our performance evaluation because it aims to reduce the BAN energy consumption and minimize the number of packets forwarded by intermediate nodes and has the same characteristic as our protocol, namely it's an energy aware routing protocol for hospital body area network communication. The evaluation is accompanied with an analysis and discussion of results.

\section{A. Performance parameters}

We evaluate two key performance metrics. Energy consumption is the average of the energy consumed by nodes participating in packets transfer from the source node to the destination node during the whole simulation. The routing overhead is measured as the number of control messages transmitted at each node during the simulation. Each message hop is counted as one separate transmission.

\section{B. Performance evaluation}

We carried out simulations to determine the effectiveness of our protocol. The principal goal of these simulations is to analyze our protocol by comparing it with other protocols, mainly EPR [18].

To evaluate ESR, we use the network simulator ns-2 [20]. Each simulation run has a duration of 400 seconds. During each simulation, constant bit rate (CBR) connections are generated; the node source produces four packets per second with a packet size of 32 bytes. The values of RREQ_Wait_Time and RREP_Wait_Time are set to 0.7 seconds. We vary the number of network BAN coordinators nodes from 8 to 16 to obtain different scenarios in an $100 \mathrm{~m} \times 100 \mathrm{~m}$ environment. The Random Waypoint model is used to simulate BAN coordinator movement; each BAN coordinator node moves with a speed randomly chosen from 0 to $3 \mathrm{~m} / \mathrm{s}$. The radio model is a shared-media radio with a nominal bit-rate of $250 \mathrm{Kbps}$ and a nominal radio range of $10 \mathrm{~m}$, which is compatible with the IEEE 802.15.4 standard. We assume that a node consumes $7 \mathrm{dbm}$ while receiving and $15 \mathrm{dbm}$ while transmitting. It was shown in [21] that no real node energy optimization can be achieved in the presence of overheating or in idle state. For this reason, the energy consumption during idle or overheating time is not considered in this model. Each simulation is carried out under a different number of network nodes and the performance metrics are obtained by averaging over 20 simulation runs from one source BAN coordinator to the static coordinator. In our simulations, we initialized the energies of the nodes randomly between 10 and 50 Joules (uniform distribution), which corresponds to the average capacity of a BAN coordinator battery. We choose $\alpha=\beta$ in order not to favour any criteria.
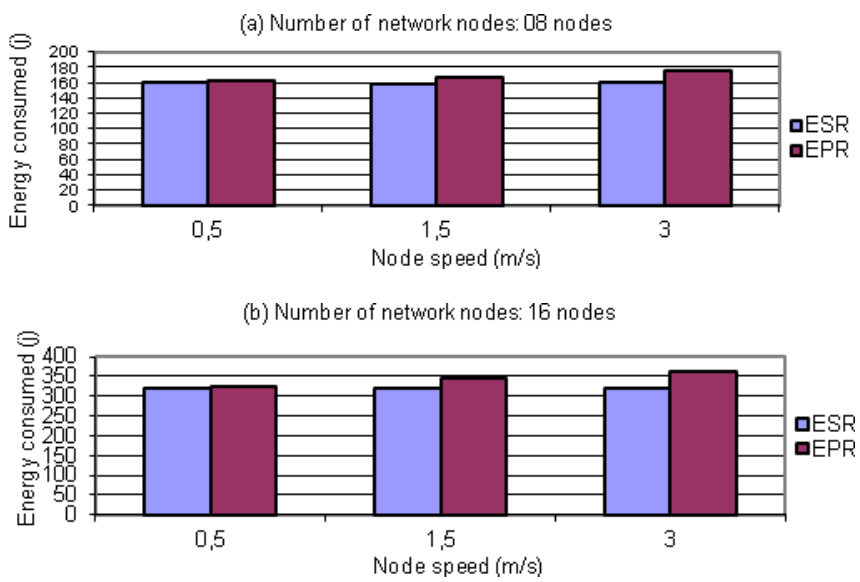

Figure 3: Energy consumed versus node speed.

Our simulations are performed in different speeds moves of nodes for different nodes number by the ESR and EPR protocols. From Figure 3 (a) we can observe that the energy consumed by our protocol ESR is less than the EPR protocol; this decrease is significant in the case shown in Figure 3 (b), the reasons is that when the maximum speed of nodes increases this favours the links instability and increases the nodes disconnections. Our protocol solves this inefficiency by selecting the more stable links for packets transmitting. ESR focuses not only on the energy capacity of a node, which can also get depleted if there is a high 
level of traffic passing through it, but also on the path stability.

(a) Number of network nodes: 08 nodes

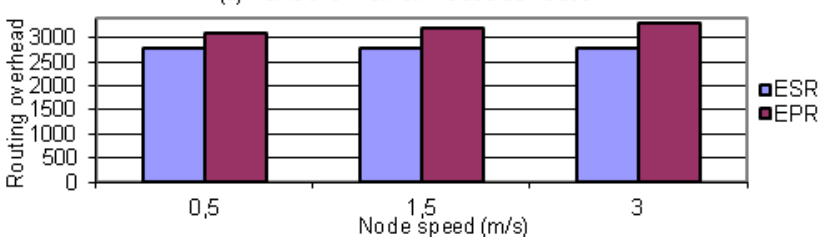

(b) Number of network nodes: 16 nodes

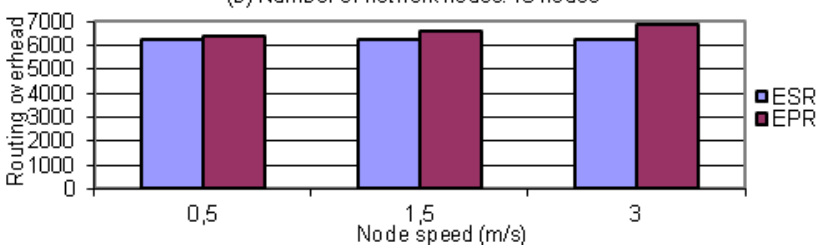

Figure 4: Routing overhead versus node speed

From Figure 4 we note that the routing control traffic is more important in the EPR. For instance, 3199 routing messages (in the case of the 8 nodes network and $1.5 \mathrm{~m} / \mathrm{s}$ for speed node) have been forwarded in the network during a $400 \mathrm{~s}$ time simulation versus 2780 for the ESR protocol. The ESR protocol generates less control traffic than the EPR protocol. The increase in our protocol performance is provided by the use of stable path which has less link fails, these fails requires the relaunch of the path discovery which increases the number of control packets. As can be seen, in all cases, ESR has a smaller number of control packets compared with EPR, which explains that the protocol ESR can assure that stable paths with higher energy are identified and selected for transmission. The ESR protocol improves the number of control packets compared with EPR.

\section{CONCLUSION}

In this paper we have proposed a new energy aware and stable routing protocol (ESR) for mobile WBAN networks. This protocol is designed for a hospital environment. Mobile WBAN networks are characterized by their dynamicity, mainly in point-to-point BAN communication: link failures and path breaks occur frequently. Moreover, the frequent changes of topology exhaust the batteries of the nodes, which decrease the network performance. The new protocol ESR uses an energy aware and stable mechanism, which exploits the residual energy and stability of nodes to select the best path for transmitting data packets. This concept improves the network performance when compared to the state of the art. Simulation results have shown that our protocol consumes less energy and minimizes the routing overhead traffic. We conclude that our protocol ESR improves the mobile WBAN network performance. This work shows some interesting possibilities where our ESR solution can be extended to include criteria for security and energy management during the path discovery process.

\section{References}

[1] M. Chen, S. Gonzalez, A. Vasilakos, H. Cao, and V. C. Leung, "Body area networks: A survey," Mobile Netw. Appl., vol. 16, pp. 171-193, Apr. 2011.

[2] E. Reusens, W. Joseph, B. Latré, B. Braem, G. Vermeeren, E. Tanghe, L. Martens, I. Moerman, C. Blondia, "Characterization of on-body communication channel and energy efficient topology design for wireless body area networks," IEEE Transactions on
Information Technology in Biomedicine, Vol 13, No. 6, pp. 933 945, 2009.

[3] A. Ehyaie, M. Hashemi, P. Khadivi, "Using relay network to increase life time in wireless body area sensor networks," in: Proc. Of WoWMoM'09, Kos, Greece, pp. 1-6, 2009.

[4] X. Liang, X. Li, Q. Shen, R. Lu, X. Lin, X. Shen, W. Zhuang, "Exploiting prediction to enable secure and reliable routing in wireless body area networks," in: Proc. of IEEE INFOCOM, Orlando, Florida, USA, pp. 388-396, 2012.

[5] Q. Zhang, K. Kortermand, R.H. Jacobsen, T.S. Toftegaard, "Reactive virtual coordinate routing protocol for body sensor networks," in: Proc. of IEEE ICC, Ottawa, Canada, pp. 3388-3393, 2012.

[6] S. Ahmed, N. Javaid, S. Yousaf, A. Ahmad, M. M. Sandhu, Z. A. Khan, N. Alrajeh, M. Imran, "Co-LAEEBA: Cooperative Link Aware and Energy Efficient Protocol for Wireless Body Area Networks," Computers in Human Behavior, 2015.

[7] L. Liang, Y. Ge, G. Feng, W. Ni and A.A.P. Wai, "A low overhead tree-based energy-efficient routing scheme for multi-hop wireless body area networks," Computer Networks, Vol.70, pp. 45-58, 2014.

[8] J. Elias, "Optimal design of energy-efficient and cost-effective wireless body area networks," Ad Hoc Networks, Vol. 13, pp. 560 574, 2014.

[9] D.S. Zois, M. Levorato and U. Mitra, "Energy-Efficient, Heterogeneous Sensor Selection for Physical Activity Detection in Wireless Body Area Networks," IEEE Transactions on signal processing, Vol.. 61, No. 7, pp. 1581-1594, 2013

[10] C.S. Lin, P.J. Chuang, "Energy-efficient two-hop extension protocol for wireless body area networks," IET Wirel. Sens. Syst., Vol. 3, No. 1, pp. 37-56, 2013.

[11] Q. Nadeem, N. Javaid, S.N. Mohammad, M.Y. Khan, S. Sarfraz, and M. Gull, "SIMPLE: Stable increased-throughput multi-hop protocol for link efficiency in wireless body area networks," IEEE Eighth international conference on broadband and wireless computing, communication and applications (BWCCA), pp. 221-226, 2013.

[12] S. Ahmed, N. Javaid, and S. Yousaf, "Co-CEStat: Cooperative critical data transmission in emergency for static wireless body area network," Journal of Basic and Applied Scientific Research, Vol. 4, No. 1, pp. 200-216, 2014.

[13] M. Maalej, S. Cherif, and H. Besbes, "QoS and energy aware cooperative routing protocol for wildfire monitoring wireless sensor networks," The Scientific World Journal, 2013.

[14] G. Cheng, R.V. Prasad, M. Jacobsson, "Packet forwarding with minimum energy consumption in body area sensor networks," in:Proc. IEEE Consumer Communications and Networking Conference (CCNC), pp. 1-6, 2010.

[15] A. Maskooki, C. B. Soh, E. Gunawan, K. S. Low, "Opportunistic routing for body area network," In Proc. IEEE Consumer Communications and Networking Conf., 2011.

[16] A. Tauqir, N. Javaid, S. Akram, A. Rao, S. N. Mohammad, "Distance Aware Relaying Energy-efficient: DARE to Monitor Patients in Multi-hop Body Area Sensor Networks," IEEE Eighth International Conference on Broadband and Wireless Computing, Communication and Applications (BWCCA), 26 July 2013.

[17] M. R. Jafri. Javaid, A. Javaid, Z.A Khan, "Maximizing the lifetime of multi-chain pegasis using sink mobility". World Applied Sciences Journal, Vol. 21, No. 9, pp. 1283-1289, 2013.

[18] Z. A. Khan, S. Sivakumar, W. Phillips, N. Aslam, "A new patient monitoring framework and Energy aware Peering Routing Protocol (EPR) for Body Area Network communication," Journal of Ambient Intelligence and Humanized Computing, Vol. 5, No. 3, pp. 409-423, June 2014.

[19] K. Hur, W-S. Sohn, , J-K. Kim, Y. Lee, "A WBAN Beacon Structure for Wireless USB Protocol Adaptation," International Journal of Multimedia and Ubiquitous Engineering, vol. 8, no. 2, pp. 143--158, March 2013.

[20] The Network Simulator ns-2, available online at http://www.isi.edu/nsnam/ns.

[21] D Kim, J J Garcia-Luna-Aceves, K Obraczka, J-C Cano, P Manzoni, "Routing mechanisms for mobile ad hoc networks based on the energy drain rate," IEEE Transactions on Mobile Computing, Vol. 2, No. 2, pp. 161-173, 2003. 